\title{
Hepatocellular carcinoma and immune therapy from a clinical perspective: where are we?
}

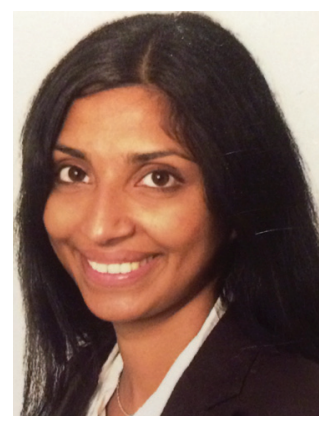

"The next few years will likely see the evaluation

in the clinic of new immune-based

combinations...”

\section{Susanna V Ulahannan ${ }^{*, 1} \&$ Austin G Duffy ${ }^{2}$}

First draft submitted: 28 July 2016; Accepted for publication: 29 July 2016;

Published online: 19 August 2016

The incidence of hepatocellular carcinoma (HCC) has been reported to continue to increase in the past decades [1], however, we and other groups have found that this might be changing in the USA $[2,3]$. In our study - based on SEER data - there seemed to be a plateau in incidence rate around 2007 , and that the rate of diagnosis in patients with smaller tumors $(<5 \mathrm{~cm})$ surpassed larger tumors around 2005. Despite these positive findings, less than $25 \%$ of patients received potentially curative treatment with liver transplantation, resection or ablation. In patients who are not candidates for curative treatments, options are limited to transcatheter arterial chemoembolization (TACE) for intermediate stage disease and, in the advanced disease setting, systemic therapy with sorafenib, the only US FDA-approved drug treatment $[4,5]$. Recently, a Phase III trial reported that regorafenib in the second-line setting improved overall survival by 2.8 months
(10.6 vs 7.8 months) when compared with placebo (HR: 0.62; $\mathrm{p}<0.02$ ) [6]. This was the first positive trial in many years and we will await further details from this study. There have been several Phase II and III trials attempting to improve the limited treatment options in HCC but unfortunately these trials have been negative [7]. Other Phase III trials that are ongoing in second-line setting include cabozantinib (NCT01908426), MET inhibitor tivantinib (NCT01755767) and the VEGFR2 inhibitor ramucirumab (NCT02435433). Thus there is an urgent need to find alternative durable and well-tolerated treatments in HCC.

There are many indications in the literature suggesting that HCC should be amenable to immune therapy. HCC has been reported to a cancer type where spontaneous tumor regressions are seen $[8]$, although this is likely due to diverse mechanisms including either immune responses or vascular

\section{KEYWORDS}

- check point inhibitors $•$ CTLA-4 inhibitor $\bullet$ hepatocellular carcinoma - immune therapy • PD-1 inhibitor - radiofrequency ablation - RFA

- TACE • transcatheter arterial chemoembolization

\section{"To further optimize outcome, combination strategies are needed to overcome tumor evasion.”}


auto-embolization by tumor [9]. HCC is characterized by chronic inflammation and immune inhibitory checkpoints appear to be contributing to immune suppression in this setting [10] and subsequently the development and progression of HCC [11,12]. The liver is unique in the required intricate balance it needs to strike between not over-reacting to the antigens absorbed by the gut and accurate immune responses and elimination of tumor antigen. Tregs and myeloid-derived suppressor cells are thought to be important in protecting the tumor from eradication by activated cytotoxic $\mathrm{CD}^{+} \mathrm{T}$ cells. High levels of Tregs in tumor have been linked to poor prognosis in HCC. There are many excellent reviews describing these multifaceted immune interactions in detail $[7,12]$. Therapeutically, blockade of the so-called immune checkpoints will only work in the setting of an ongoing antitumor immune response. In patients with advanced HCC and concurrent hepatitis $\mathrm{C}$ virus, a Phase II trial evaluated tremelimumab, a CTLA-4 inhibitor. The trial showed partial response rate of $17.6 \%$, stable disease $76.4 \%$ and time to progression was 6.5 months, providing proof of principle that alleviating the suppression of antitumor immunity can be effective at least in a subset of patients. Interestingly, a decrease in viral load was observed, presumably due to enhanced T-cell response [13]. With regard to the other major 'checkpoint', PD-1, promising interim data for nivolumab was reported at the annual ASCO meeting 2016 by El-Khoueiry et al. [14]; at the time of analysis 48 patients were evaluable for response, with an objective response rate of $15 \%$, including 3 patients with complete response and $50 \%$ with stable disease, with median overall survival of 15.1 months, an impressive result for the second-line setting. Notably, response did not appear to be related to PD-L1 expression or hepatitis infection status. The treatment was tolerable and in a subset of patients the clinical benefit was durable. To further optimize outcome, combination strategies are needed to overcome tumor evasion. The combination of anti-CTLA 4 and anti PD-1 dual blockade was FDA approved in September 2015 in melanoma [15]. In view of the early data seen in HCC with single-agent check point inhibitor, combination therapy appears the logical next step, and this is currently underway in the clinic (NCT02519348). What about other strategies? One of the great potentials of immune modulating therapies is that they can dovetail with standard modalities, enhancing their effects on the immune system. For example, both TACE and radiofrequency ablation (RFA) have been shown to stimulate systemic antitumor immunity, presumably from release of tumor antigen [16-19] and hold promise for combining with immune modulation. Indeed, this approach has been shown to be safe, feasible and associated with encouraging clinical activity in an advanced patient population [20]. Most of the trials underway are in advanced HCC second-line setting, but there is also a Phase III study ongoing comparing nivolumab with sorafenib as first-line therapy in advanced HCC, the aim is to enroll 726 patients (NCT02576509). This is an exciting time for immune-based approaches in HCC and the early data suggest that a subset of patients are benefiting, with durable responses, and good tolerability, in a disease with few treatment options beyond local therapies. The next few years will likely see the evaluation in the clinic of new immune-based combinations, either paired with each other or with standard treatment modalities, enhancing and magnifying their effects.

Financial \& competing interests disclosure The authors have no relevant affiliations or financial involvement with any organization or entity with a financial interest in or financial conflict with the subject matter or materials discussed in the manuscript. This includes employment, consultancies, honoraria, stock ownership or options, expert testimony, grants or patents received or pending, or royalties.

No writing assistance was utilized in the production of this manuscript.

\section{References}

1 Torre LA, Bray F, Siegel RL et al. Global cancer statistics, 2012. CA Cancer J. Clin. 65, 87-108 (2015).

2 Ulahannan SV, Duffy AG, McNeel TS et al. Earlier presentation and application of curative treatments in hepatocellular carcinoma. Hepatology 60, 1637-1644 (2014).
3 Njei B, Rotman Y, Ditah I et al. Emerging trends in hepatocellular carcinoma incidence and mortality. Hepatology 61, 191-199 (2015).

4 Bruix J, Reig M, Sherman M. Evidence-based diagnosis, staging, and treatment of patients with hepatocellular carcinoma. Gastroenterology 150, 835-853 (2016).
5 Llovet JM, Ricci S, Mazzaferro V et al. Sorafenib in advanced hepatocellular carcinoma. N. Engl. J. Med. 359, 378-390 (2008).

6 Bruix J MP, Merle P, Granito A et al. Efficacy and safety of regorafenib versus placebo in patients with HCC progressing on sorafenib: results of the international, randomized 
Phase 3 RESORCE trial. Presented at: The World Congress on GI Cancer. Barcelona, Spain, 28 June-2 July 2016.

7 Greten TF, Wang XW, Korangy F. Current concepts of immune based treatments for patients with HCC: from basic science to novel treatment approaches. Gut 64, 842-848 (2015).

$8 \quad$ Iwanaga T. [Studies on cases of spontaneous regression of cancer in Japan in 2011, and of hepatic carcinoma, lung cancer and pulmonary metastases in the world between 2006 and 2011]. Gan To Kagaku Ryoho 40, 1475-1487 (2013).

9 Huz JI, Melis M, Sarpel U. Spontaneous regression of hepatocellular carcinoma is most often associated with tumour hypoxia or a systemic inflammatory response. $H P B$ (Oxford) 14, 500-505 (2012).

10 Kassel R, Cruise MW, Iezzoni JC et al. Chronically inflamed livers up-regulate expression of inhibitory B7 family members. Hepatology 50, 1625-1637 (2009).

11 Shi F, Shi M, Zeng Z et al. PD-1 and PD-L1 upregulation promotes $\mathrm{CD} 8\left(^{+}\right) \mathrm{T}$-cell apoptosis and postoperative recurrence in hepatocellular carcinoma patients. Int. J. Cancer 128, 887-896 (2011).
12 Makarova-Rusher OV, Medina-Echeverz J, Duffy AG et al. The yin and yang of evasion and immune activation in HCC. J. Hepatol. 62, 1420-1429 (2015).

13 Sangro B, Gomez-Martin C, de la Mata M et al. A clinical trial of CTLA-4 blockade with tremelimumab in patients with hepatocellular carcinoma and chronic hepatitis C. J. Hepatol. 59, 81-88 (2013).

14 El-Khoueiry AB, Sangro B, Yau TC et al. Phase I/II safety and antitumor activity of nivolumab (nivo) in patients (pts) with advanced hepatocellular carcinoma (HCC): interim analysis of the CheckMate-040 dose escalation study. J. Clin. Oncol. 34 (15 Suppl.), Abstract 4012 (2016).

15 Larkin J, Chiarion-Sileni V, Gonzalez R et al. Combined nivolumab and ipilimumab or monotherapy in untreated melanoma. N. Engl. J. Med. 373, 23-34 (2015).

16 Nobuoka D, Motomura Y, Shirakawa H et al. Radiofrequency ablation for hepatocellular carcinoma induces glypican-3 peptide-specific cytotoxic T lymphocytes. Int. J. Oncol. 40, 63-70 (2012).

17 Zerbini A, Pilli M, Laccabue D et al. Radiofrequency thermal ablation for hepatocellular carcinoma stimulates autologous NK-cell response. Gastroenterology 138, 1931-1942 (2010).

18 Ayaru L, Pereira SP, Alisa A et al. Unmasking of alpha-fetoprotein-specific $\mathrm{CD} 4\left(^{+}\right) \mathrm{T}$ cell responses in hepatocellular carcinoma patients undergoing embolization. J. Immunol. 178, 1914-1922 (2007).

19 Hiroishi K, Eguchi J, Baba T et al. Strong CD8 $\left(^{+}\right)$T-cell responses against tumorassociated antigens prolong the recurrence-free interval after tumor treatment in patients with hepatocellular carcinoma. J. Gastroenterol. 45, 451-458 (2010).

20 Duffy AG, Makarova-Rusher OV, Pratt D et al. Tremelimumab: a monoclonal antibody against CTLA- 4 - in combination with subtotal ablation (trans catheter arterial chemoembolization (TACE), radiofrequency ablation (RFA) or cryoablation) in patients with hepatocellular carcinoma (HCC) and biliary tract carcinoma (BTC). J. Clin. Oncol. 34(15 Suppl.), Abstract 4073 (2016). 